\title{
Geographic Information Realization of Highway Slope Database based on VB and JAVAScript
}

\author{
Zhijie SUN ${ }^{1, a}$, Ziyang ZHAO ${ }^{1, b}$ and Lin $\mathrm{MA}^{1, \mathrm{c}}$ \\ Key Lab of Highway Construction \& Maintenance Technology in Loess Region, Shanxi \\ Transportation Research Institute, Shanxi, Taiyuan, 030006

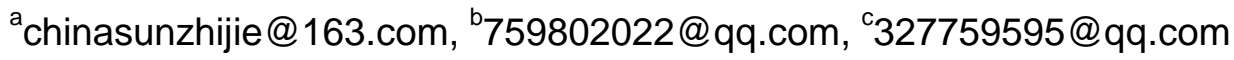

\begin{abstract}
Keywords: Slope database; Geographic Information; VB; JAVAScript
Abstract. The database management system of highway slope database can improve the level of data processing, regulate the data collection and improve the working efficiency. Visual Basic as the main development process, with the help of development features of Baidu map API, and use of JAVAScript language to achieve display slope in map by the VB framework of the visual window. Which can provide a reference for the slope engineering and similar projects in visualization information.
\end{abstract}

\section{Introduction}

There are large number of highway slopes in mountainous areas, traditional method of slopes data and data management is very inconvenient. But in the slope analysis, the slope instability mechanism and the slope stability evaluation to involve a lot of slope sample and its parameters, need to support the slope database and its management system. With the help of computer and network, using of existing technology and conditions, to improve the data processing level through the database management system, to improve the efficiency of data collection and improve the working efficiency is a practical significance issue.

Make full use of existing equipment resources based on the consideration, combined with the actual situation of slope on Jixian-Hejin Expressway, Visual Basic as the main development process, with the help of development features of Baidu map API, and use of JAVAScript language to achieve display and research slope in map by the VB framework of the visual window. The slope database system can provide a reference for the slope engineering and similar projects in visualization information.

\section{System Requirement Analysis}

The database management system of highway slopes is a software for information management which is specially designed for the management of the highway slopes. The system should have the basic information include picture information of slope data, it functions of add, delete, modify and reporting the information of the slope, also can carry out the multi-condition query and statistical function of the slope.

Different from other slope database management system, the system also has the function of the slope input with GPS coordinate information in map and the precision display function of the GPS coordinates of the slope.

Data collection and update of the slope database through the design of survey data, field image information collection and GPS coordinates. Collecting information of slope is in real time, system and accurate, which can provide the necessary data support for the data analysis of technical personnel, management personnel maintenance and rescue work.

\section{Function Module Design of Slope Map Retrieval}

The main function of the slope map retrieval interface is based on the height of the slope and the length of the query, and display the distribution of the slope in webbroswer control of VB program to 
meet the retrieval conditions. The operation object of the slope map query interface is shown in table 1 .

Table 1 Description of the slope map query interface

\begin{tabular}{ccc}
\hline No & Operational Objectives & Instruction \\
\hline 1 & List Box-Province & Select the province name from the drop-down list \\
2 & List Box-Highway & Select the highway name from the drop-down list \\
3 & List Box-Stratigraphic combination & Selection of the strata in the drop down list \\
4 & Text Box-Slope height & Input height range of slope \\
5 & Text Box-Slope length & Input length range of slope \\
6 & Button-Search & Display the slopes in map to meet the search terms \\
\hline
\end{tabular}

\section{Realization of Slope Map Retrieval Information}

Webbroswer control of Visual Basic can easily display the content of the web page, and the Baidu map API can provide powerful development function which it can retrieval and display the slope information in Baidu map conditions. Through the JAVAScript language to achieve the call of the Baidu map API, and then through the webbroswer control of Visual Basic to achieve the map of the slope of the search and display.

The partial code of slope map information retrieval is as follows.

$<$ label for="type1">Slope Height $></$ label $><$ input id="keyword1" type="text" style="width:50px;" value="0"/>

$<$ label for="type2" $>\quad$ Slope Length $></$ label $><$ input id="keyword2" type="text" style="width:50px;" value="0"/>

<input type="button" onclick="findside();;" value="Search" />

function findside()

\{

var area1 = document.getElementById("keyword1").value;

var area2 = document.getElementById("keyword2").value;

var map = new BMap.Map("map");

map.enableScrollWheelZoom(true);

var point = new BMap.Point $(111.361992 \quad$, 38.648004);

map.centerAndZoom(point, 10);

var point = new Array(); // An array about the latitude and longitude of the label points.

var marker = new Array(); // An array about the object of the label points.

var info = new $\operatorname{Array}() ; / /$ An array about tip information window.

var sum_p0 $=0 ; / /$ Coordinate of $\mathrm{p} 0$.

var sum_p1=0;// Coordinate of $\mathrm{p} 1$.

var $\mathrm{j}=0 ; / /$ Counter

for ( $\operatorname{var} \mathrm{i}=0 ; \mathrm{i}<$ markerArr.length; $\mathrm{i}++)\{$

var address $=$ parseInt (markerArr[i].address); // Extract the height of slope.

var tel = parseInt(markerArr[i].tel); // Extract the Length of slope.

if (address > parseInt(area1) \&\& tel > parseInt(area2) \{

$\mathrm{j}=\mathrm{j}+1$;

var $\mathrm{p} 0=$ markerArr[i].point.split(",")[0]; //

var p1 = markerArr[i].point.split(",")[1]; // Extract the latitude and longitude of the

map points.

point $[\mathrm{i}]=$ new window.BMap.Point $(\mathrm{p} 0, \mathrm{p} 1)$; // Generate a new map points.

sum_p0=sum_p0+parseInt (p0);

sum_p1=sum_p1+parseInt $(\mathrm{p} 1)$; 
with the map point above.

marker[i] = new window.BMap.Marker(point[i]); // Generating the marking point map.addOverlay(marker[i]);

var label = new window.BMap.Label(markerArr[i].title, \{ offset: new window.BMap.Size(20, -10) \});

marker[i].setLabel(label); info[i] $\quad=\quad$ new window.BMap.InfoWindow $("<\mathrm{p}$ style='font-size:12px;lineheight:1.8em;'>" + markerArr[i].title + "</br>Height of slope:" + markerArr[i].address + "</br $>$ Length of slope:" + markerArr[i].tel $+"</$ br $></ p>")$; // Create information windows.

\}

sum_p0=sum_p0/j;

sum_p1=sum_p1/j;

//alert("Longitude:" + sum_p0 + ", " + " latitude: " + sum_p1 + ", " + "Number:" + j);

map.panTo(new BMap.Point(sum_p0,sum_p1))

\}

\section{Effect of slope map retrieval}

Display effect in map according to the search terms are shown in Figure 1.

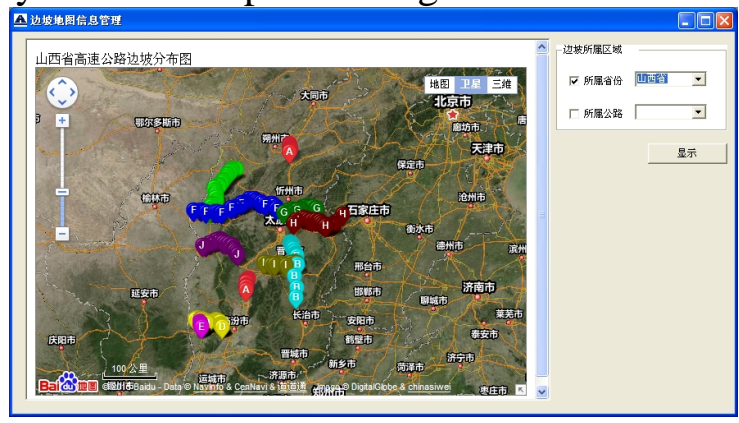

(a)Multiple routes

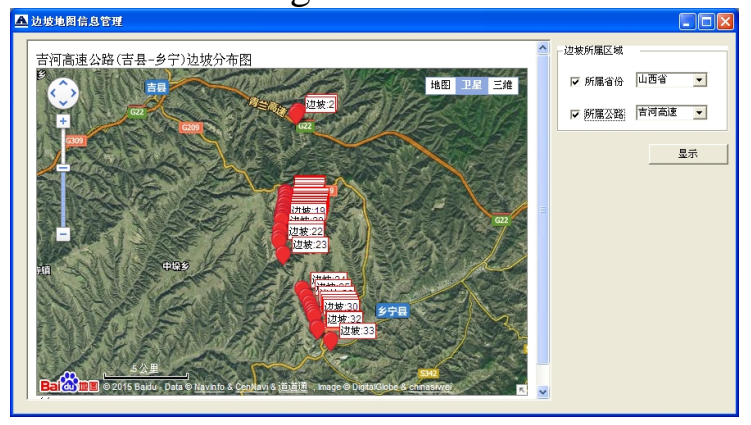

(b)A route

Fig.1 Display effect of slope map retrieval

\section{Conclusions}

The database management system of slope uses Basic Visual and JAVAScript language, through the Baidu API to achieve the map search and display of the slope. In the later period, the 3D visualization and the 3D soil information modeling of the slope can developed. It can also provide the geometric and stratigraphic model for the finite element analysis software, which can improve the accuracy and efficiency of the numerical analysis of slopes.

\section{References}

[1] WEI-Dong MO: Xi'an Jiao Tong University press, (2003).

[2] Hong-lin ZHANG: People's Posts and Telecommunications Press, (2002).

[3] Xiu-zhong LI, Yong-qin RUI and Cheng-ming LU: HIGHWAY, Vol. (11) (2005), p. 98-101.

[4] Wu XU and He-Lin FU: TECHNOLOGICAL DEVELOPMENT OF ENTERPRISE. Vol. 33(7) (2014), p. 23-25. 